\title{
Inhalt von Band 26.
}

\author{
Heft 1 nnd 2.
}

Ausgegeben Im Junl 1934 .

Selte

G. Herzberg und $\boldsymbol{H}$. Sponer, Uber die Dissoziationswärme des Stickstoffmoleküls. (Mit 1 Figur im Text) . . . . . . . . . . . . . . . . . . 1

Paul Günther und Günther Cohn, Die Anregung von Gasreaktionen durch Ionen. (Mit I Figur im Tert) . . . . . . . . . . . . . . . . . . .

M. A. Govinda Rau und B. N. Narayanaroamy, Die Bestimmung von Dipolmomenten nach der Methode der verdünnten Lösungen. I. und II. Teil. (Mit 6 Figuren im Text) . . . . . . . . . . . . . . . . . . 23

P. Feldmann und A. Stern, Zur Photolyse der Silberhalogenide . . . . . . . 45

Alfons Krause, Uber Ferromagnetismus und Struktur des Eisen (II, III)-oxyds. (Mit 3 Figuren im Text) ................ 58

G. Briegleb, Zur Theorie der Molekülverbindungen organisoher Nitrokörper. (Mit 5 Figuren im Text). . . . . . . . . . . . . . . . . . . .

Gerhard Brauer, Ein liobtelektrisches Photometer für reaktionskinetische Messungen. (Mit l Figur im Tert). . . . . . . . . . . . . . . . . .

W. Geffcken und D. Price, Zur Frage der Konzentrationsabhängigkeit des soheinbaren Molvolumens und der scheinbaren Molrefraktion in verdünaten Lösungen. (Mit 5 Figuren im Text) . . . . . . . . . . . . . .

I. N. Stranski und $R$. Kaischew, Uber den Mechanismus des Gleichgewichtes kleiner Kriställohen. (Mit 2 Figuren im Text) . . . . . . . . . . 100

R. Kaischew und I. N. Stranski, Uber den Mechanismus des Gleiohgewiohts kleiner Kriställchen. II . . . . . . . . . . . . . . . . . . . . . . 114

Alfred Coehn und Bruno Wolfgang May, Uber das Ausbleiben der Photolyse extrem getrockneten Kohlendioxyds. (Mit 3 Figuren im Text) . . . . 117

H. Epstein und W. Sleiner, Messung des RamaN-Effektes bei tiefen Temperaturen. (Mit 8 Figuren im Text). . . . . . . . . . . . 131

Heft 3.

Ausgegeben Im Jull 1034.

Richard Reinicke, Bestimmt gerichtete, schwache Nebenvalenzkräfte als die wirklichen Farbträger (Chromophore). (Mit 11 Figuren im Text) . . . . . 159

J. A. Chrigliansen und Inger Wulff, Untersuchungen über das LieszasanaPhänomen. I. Versuch zu einer Theorie von J. A. Christiansen . . . 187

Sten Friberg, Uber eine Gleichförmigkeit in der Dispersion flüssiger und gasförmiger Kohlenwasserstoffe. (Mit 2 Figuren im Tert) . . . . . . . . 195

Karl F. Herzfeld und Maria Qoeppert-Mayer, Das Verhalten von in Palladium gelöstem Wasserstoff. . . . . . . . . . . . . . . 203

K. W. F. Kohlrausch und F. Köppl, Studien zum RAMax-Effekt. Mitteilung XXXII. Die Raman-Spektren der Paraffine. (Mit 3 Figuren im Text) 209

E. Pohland, Eine handliohe Röntgen-Vacuumkamera für beliebige tiefe Temperaturen. (Mit 3 Figuren im Toxt) . . . . . . . . . . . . . . . . 238

M. Straumanis, Das Wachstum von Metallkristallen im Metalldampf. III. (Mit 7 Figuren im T'ext) . . . . . . . . . . . . . . . 246

Heft 4 und 5 .

Ausgegeben im August 1034.

J. C. Ghosh, D. S. Narayana Murthi und D. N. Das Gupta, Untersuchungen über die photosensibilisierende Wirkung ron Jod. Die isomere Umwandlung von Allo-cinnamyliden-essigsäure in die Normalform. Teil I . . . . 255 
J. C. Ghosh und D. S. Narayana Murthi, Untersuchungen über die photosensibilislerende Wirkung von Jod. Die isomere Omwandlung von Allo-cinnamyliden-essigsäure in die Normalform. Teil II . . . . . . . . . . . 267

E. A. Moeleyn-Hughes, Die katalytisohe Wirksamkeit des Deuteriumions $\left(D, O^{+}\right), 272$

E. A. Moelwoyn-Hughes, Uber den Temperaturkoeffizienten der Rohrzuckerinversion.

Hua-Chih Cheng, Studien zum Raman-Effekt. Mitteilung XXXV. Die RamanSpektren einiger Acetyl - und Acetonitrilverbindungen. (Mit 3 Figuren im Text) . . . . . . . . . . . . . . . . . . . . . . . . . . . 288

$A$. Eucken und $B$. Ahrens, Die Normalschwingungen des Schwefelhezafluorids. (Teilweise unter Mitwirkung von E. Bartholome und L. Bewrloova.) (Mit 4 Figuren im Tert) . . . . . . . . . . . . . . . . . . . . . 297

I. N. Stranski und R. Kaiochew, Uber den Mechanismus des Gleicbgewichts kleiner Kriställohen. III . . . . . . . . . . . . . . . . . . . . . . 312

R. Kaischew und $I$. N. Stranski, Zur kinetischen Ableitung der Keimbildungsgeschwindigkeit. (Mit 1 Figur im Text) . . . . . . . . . . . . . . . 317

J. A. A. Ketelaar, Des elektrische Leitvermögen des $\mathrm{Ag}_{2} \mathrm{HgJ}_{4}$. Ein Beitrag zur Frage nach dem Leitungomechanismus in den gatleitenden festen Stoffen. (Mit 2 Figuren im Tert) . . . . . . . . . . . . . . . . . . . . 327

R. Klar, Uber die Austausohbarkeit von Wasserstoffatomen in organischen Verbindungen mit den Wasserstoffatomen des Wassers. . . . . . . . . 335

$U$. Dehlinger, Stetiger Ubergang und kritischer Punkt zwischen zwei festen Phasen. (Mit 5 Figuren im Text). . . . . . . . . . . . . . . . . 343

F. Strassmann, Untersuchungen über Oberflächengrösse und Gitteränderungen kristallisierter Salze nach der Emsniermethode von HAHN. (Mit 1 Figur im Text). . . . . . . . . . . . . . . . . . . . . . . . . 353

F. Strassmann, Untersuchungen über den Zusammenhang zwisohen Gitterstruktur und Gasdurchlässigkeit organiscber Salze nach der Emaniermethode von HaHs . . . . . . . . . . . . . . . . . . . . . . . . . . 362

S. Götzky und Paul Günther, Die Anregung der Chlorknallgasreaktion durch Röntgenstrahlen . . . . . . . . . . . . . . . . . . . . . . . . 373

Dan Rüdulescu und Octavian Jula, Beiträge zur Bestimmung der Abstufung der Polarität des Aminstickstoffes in den organischen Verbindangen. (V orläufige Mitteilung). . . . . . . . . . . . . . . . . . . . . 390

Dan Rádulescu und Octavian Julia, Uber die Rolle der Polarität der $N$-Atome bei der Bildung der farbigen Polynitrokomplexe der Benzolreihe . . . 395

Heft 6.

Ausgegeben Im September 1934.

Hans Reischauer, Uber die Adsorption von Sauerstoff und Wasserstoff an kom. paktem Platin. (Mit 5 Figuren im Tert) . . . . . . . . . . . . . . 399

Ernst Schaaff, Uber den Einfluss von Gasen auf den lichtelektrischen Effekt des Platins. (Mit 8 Figuren im Text). . . . . . . . . . . . . . . . 413

G. Karagunis und $G$. Drikos, Zur Stereochemie der freien Triarylmethylradikale. Eine total asymmetrische Synthese. (Mit 4 Figuren im Text) . . . 428

$Y$. Go und $O$. Kratky, Röntgenographisohe Studien an Choleinsäuren. (Mit 1 Figur im Text) . . . . . . . . . . . . . . . . . . . 439

H.J. Schumacher und $\boldsymbol{K}$.Wolff, Die durch belichtetes Chlor sensibilisierte Phosgenbildung aus Chloroform und Sauerstoff. (Mit 1 Figur im Text) 453

A. Eucken und $F$. Sauter, Uber die intramolekularen Kräfte in Oktaedermolekeln,

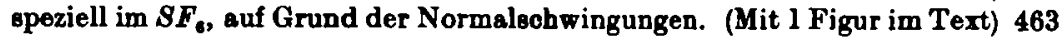

